Jasmonates: biosynthesis, perception and signal transduction

Gareth Griffiths

Energy and Bioproducts Research Institute, Chemical Engineering \& Applied Chemistry, Aston University, Birmingham, B4 7ET, UK

Email: g.griffiths@aston.ac.uk

Tel: +44(0)121 2043415 


\title{
Jasmonates: Biosynthesis, perception and signal transduction
}

\begin{abstract}
Jasmonates are physiologically important molecules involved in a wide range of plant responses from growth, flowering, senescence to defence against abiotic and biotic stress. They are rapidly synthesised from a-linolenic acid $\left(\mathrm{C} 18: 3 \Delta^{9,12,15}\right)$ by a process of oxidation, cyclisation and acyl chain shortening involving co-operation between the chloroplast and peroxisome. The active form of JA is the isoleucine conjugate, JA-lle, which is synthesised in the cytoplasm. Other active metabolites of JA include the airborne signalling molecules, methyl jasmonate (Me-JA) and cis-jasmone (CJ) which act as inter-plant signalling molecules activating defensive genes encoding proteins and secondary compounds such anthocyanins and alkaloids. One of the key defensive metabolites in many plants is a protease inhibitor that inactivates the protein digestive capabilities of insects, thereby, reducing their growth. The receptor for JA-Ile is a ubiquitin ligase termed SCFCoi1 that targets the repressor protein Jasmonate Zim domain (JAZ) for degradation in the $26 \mathrm{~S}$ proteasome. Removal of JAZ allows other transcription factors to activate the $\mathrm{JA}$ response. The levels of JA-lle are controlled through catabolism by hydroxylating enzymes of the cytochrome P450 family. The JAZ proteins act as metabolic hubs and play key roles in crosstalk with other phytohormone signalling pathways in co-ordinating genome wide responses. Specific subsets of JAZ proteins are involved in regulating different response outcomes such as growth inhibition versus biotic stress responses. Understanding the molecular circuits that control plant responses to pests and pathogens is a necessary pre-requisite to engineering plants with enhanced resilience to biotic challenges for improved agricultural yields.
\end{abstract}

\section{Introduction}

\section{Background}

Jasmonates (JAs) are lipid derived phytohormones involved in plant responses to abiotic and biotic stress as well as playing key roles in a wide range of plant developmental processes (1). The biosynthetic pathway for JA was the initial focus of research in this area and the key enzymes have been characterised and cloned over the past 25 years. Recently, much of the attention has been involved in the identification and subsequent characterisation of the receptor complex and its regulatory elements (2). This brief review will cover the key findings of the research on 1). Biosynthesis of JAs 2). The JA receptor and its regulation by transcription factors and 3). how JA interacts with other signalling pathways. The review will conclude with the future potential of exploiting the JA response for improved crop protection against pests and pathogens.

\section{Oxylipin pathways}

It should be pointed out that while JA signalling is the focus of the current review it is only one of several pathways that utilise oxygenated fatty acids in plant responses to pests and pathogens $(3,4)$ A vast array of oxygenated lipid-based signalling/antimicrobial molecules have been characterised in plants and several metabolic pathways have been elucidated. These include the dioxygenation of $\alpha-$ 
linolenic acid by an $\alpha$-dioxygenase to produce 2-hydroperoxylinolenic acid (5) and the formation of 9- and 13-hydroperoxylinolenic acid (HPL) by isozymes of lipoxygenase (4). The enzyme hydroperoxide lyase (6) cleaves both 9- and 13-HPLs to generate products such as nonenal, hexenal and traumatic acid. Peroxygenases act on 9- and 13-HPLs to form epoxy alcohols and polyhydroxy fatty acids (3) and divinyl ether synthase inserts an ether linkage into the acyl chain (7).The products generated by these enzymes are antimicrobial (8) or else influence plantherbivore/microbe interactions $(9,10)$. However, here we will focus on the metabolic pathway involved in the biosynthesis of JAs.

\section{Biosynthesis of Jasmonates.}

\section{Chloroplast-based reactions (Fig 1a and 1b).}

Jasmonic acid is derived from the $\omega-3$ fatty acid, $\alpha$-linolenic acid (ALA, C18:3 $3^{\Delta 9,12,15}$ ) which is a major component of leaf lipids, predominantly located in the galactolipids, monogalactosyldiacylglycerol (MGDG) and digalactosyldiacylglycerol (DGDG) of the chloroplast. The starter substrate for this pathway is unclear and ALA esterified to the galactolipid may be oxygenated (6) by a 13-lipoxygenase (13-LOX; Fig 1a) a non-haem iron sulphur protein. It is also possible that the ALA is first hydrolysed by a lipase from the parent lipid and then converted to 13-hydroperoxylinolenic acid (18:3$\mathrm{OOH}$; Fig1a) (11).

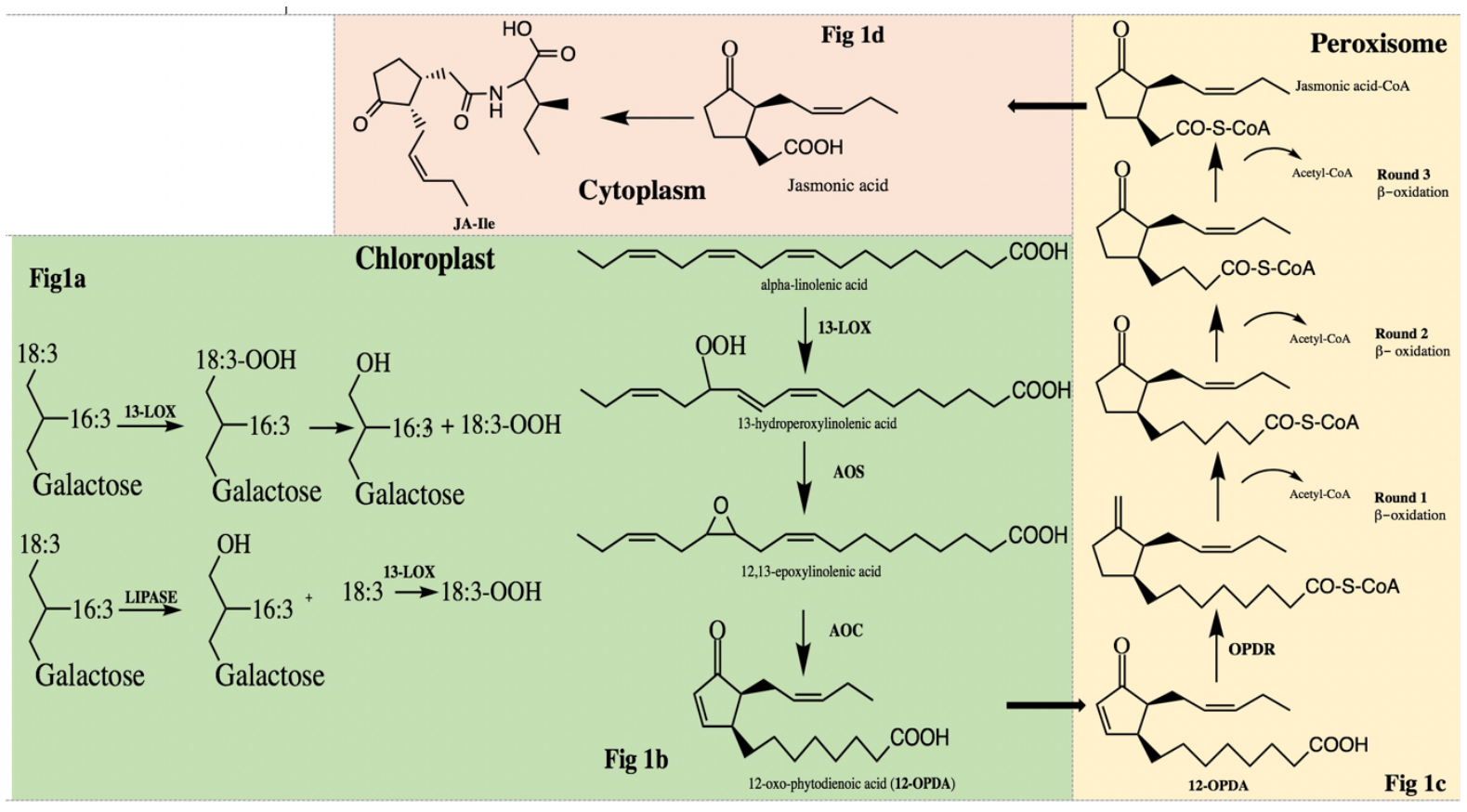


Fig. 1. Biosynthesis of Jasmonic acid.

a). The precursor for jasmonic acid (JA) is alpha-linolenic acid (18:3, ALA). It can be metabolised either directly on the galactolipid by a 13-lipoxygenase (13-LOX) or else it can be liberated by lipase hydrolysis and then the unesterified fatty acid is converted by 13-LOX to the hydroperoxy derivative (18:3-OOH). Roughanic acid (16:3) is also converted to dinor-oxo-phytodienoic acid (dnOPDA) either directly on the galctolipid and/or as the unesterified fatty acid (not illustrated) b). ALA is acted upon by a 13-LOX to generate the 13-hydroperoxylinolenic acid. A transient epoxy intermediate is then formed by the allene oxide synthesis (AOS) and this then undergoes cyclisation by the enzyme allene oxide cyclase (AOC) to generate 12-oxo-phytodienoic acid(12-OPDA) c). 12-OPDA is exported to the peroxisome where it undergoes a reduction of the cyclopentenone ring and then a series of $\beta$ oxidation reactions to generate jasmonic acid $(\mathrm{JA})$. d). JA is conjugated with isoleucine in the cytoplasm.

An analogous set of reactions can also operate on the trienoic acid $16: 3\left(\Delta^{7,10,13}\right)$ to generate a variant of jasmonic acid, termed dinor-JA (dnOPDA). Recently, extraplastidic ALA has been shown to enhance the JA defensive response raising further questions about the origins of the acyl substrate and suggesting the involvement of endoplasmic reticulum-based phospholipids such as phosphatidylcholine and/or phosphatidylethanolamine (12).

The next enzyme in the pathway is the allene oxide synthase (AOS), a cytochrome P450 enzyme of the CYP74A family that converts 18:3-OOH to 12,13-epoxylinolenic acid a transient intermediate that is then rapidly cyclised to cis $(+)-12-0 x 0-$ phytodienoic acid (OPDA) by allene oxide cyclase (AOC)(4)(Fig. 1b). In Arabidopsis a single gene encodes the AOS enzyme while there are four genes for the cyclase (AOC1-4) that appears to control different functions. The data suggest a putative regulatory mechanism of temporal and spatial fine-tuning in JA formation by differential expression and via possible heteromerisation of the four AOCs (13). Both OPDA and (7S, 11S)-10-oxo dinor phytodienoic acid (dn-OPDA) have been identified acylated at both sn-1 and sn-2 positions of galactolipids. In Arabidopsis, the levels of these cyclo-oxylipin galactolipids increase following wounding. In MGDG, OPDA was first detected at the sn-1 position and 16:3 at the sn-2. Two additional MGDG derivatives, termed arabidopsides $A$ and $B$ and two DGDG-derived (arabidopsides $C$ and $D$ have been identified (see review (3).

Detailed analysis of the galactolipid profiles of Arabidopsis following wounding reveal that there are some 167 molecular species present and a summary of the major classes are given in Table 1 . The results reveal that $63 \%$ of the galactolipids are of prokaryotic origin, i.e. synthesised within the chloroplast while the remaining proportion (37\%) is eukaryotic and imported into the organelle. The level of oxidation of the acyl chains raises rapidly following wounding and after 30 min account for almost $30 \%$ of the acyl chains in these lipids. Lipoxygenase catalysed oxidation of 16:3 gives rise to dnOPDA while 18:3 forms OPDA which is metabolised to JA. Of the oxidised galactolipids identified following wounding the major molecular species were OPDA/dnOPDA (194 $\left.\mathrm{nmol} \mathrm{g}^{-1}\right)$ with either OPDA/16:3 or 18:3/dnOPDA $\left(72 \mathrm{noml} \mathrm{g}^{-1}\right.$ ) while OPDA/OPDA was $47 \mathrm{nmol} \mathrm{g}^{-1}$ (Table 1). Since the levels of unesterified fatty acids and oxylipins derived from them are low the data suggest that the OPDA and dnOPDA are synthesised in situ on the galactolipids. 


\begin{tabular}{|l|l|l|l|}
\hline Prokaryotic & $\begin{array}{l}\text { Carbons:double } \\
\text { bonds }\end{array}$ & $\begin{array}{l}\text { Example Molecular } \\
\text { species }\end{array}$ & $\begin{array}{l}\text { Amount } \\
\mathrm{nmol} \mathrm{g}^{-1} \mathrm{fw} \\
30 \mathrm{~min}^{-1}\end{array}$ \\
\hline & $34: 6$ & $18: 3 / 16: 3$ & 738 \\
\hline & $34: 5$ & $18: 3 / 16: 2$ & 215 \\
\hline & $34: 4$ & $18: 3 / 16: 1$ & 221 \\
\hline & $34: 3$ & $18: 3 / 16: 0$ & 87 \\
\hline & $34: 2$ & $18: 2 / 16: 0$ & 47 \\
\hline & & & \\
\hline Eukaryotic & $36: 6$ & $18: 3 / 18: 3$ & 512 \\
\hline & $36: 5$ & $18: 3 / 18: 2$ & 174 \\
\hline & $36: 4$ & $18: 3 / 18: 1$ & 62 \\
\hline & $36: 3$ & $18: 0 / 18: 3$ & 12 \\
\hline & $36: 2$ & $18: 0 / 18: 2$ & 3 \\
\hline
\end{tabular}

Table 1. Molecular species of non-oxidised galactolipids present in Arabidopsis 30 min post-wounding (data re-worked from [14]

The pools of galactoplipid are classified as either prokaryotic (i.e. containing a C16 acyl chain in the molecule at the sn-2 position) or as eukaryotic which only contain $\mathrm{C} 18$ chain length acyl groups. As an example in $\mathrm{C} 34: 5, \mathrm{C} 16+\mathrm{C} 18=\mathrm{C} 34$. The number of double bonds (5) could be derived from either combination of $18: 3 / 16: 2$ or $18: 2 / 16: 3$.

It has been suggested that arabidopsides may serve a dual function as antipathogenic substances and as storage compounds that allow the slow release of free jasmonates (14). The presence of both OPDA and dOPDA in galactolipids to quite high levels (7-8\% of total leaf lipid content) in Arabidopsis appear to play roles in race specific resistance against bacterial pathogens (15). 12-oxo-PDA triggers a distinct set of genes and plays a role in wound-induced gene expression in Arabidopsis (16). However the widespread occurrence of glycolipid bound precursors for JA biosynthesis is questionable and may be restricted to a number of species (17) as they are not detected in other well studied species such as tomato and potato. Interestingly, JA shows little direct antimicrobial activity against a range of common disease-forming fungi and bacteria whereas OPDA is a potent antimicrobial compound which contains an $\alpha, \beta$-unsaturated carbonyl group (18). Studies on the structural basis for this have been made and the C8 chain arm combined with a cyclopentene ring was shown to be the most effective molecule in antimicrobial tests (18). OPDA is exported from the chloroplast to the peroxisome and may involve specific transporter proteins (19).

\section{Peroxisomal reactions (Fig 1c)}

The cyclopentenone ring of OPDA is reduced by the OPDA reductase (OPDR) to the cyclopentanone (Fig 1c). In nature only (9S,13S)-OPDA [cis-(+)-OPDA] occurs which is one of the four possible diastereomers. This stereospecific structure at the ring is established by $A O C$ and is kept in the subsequent reactions. The final product is one of four possible diastereomers, the $(3 R, 7 S)-\mathrm{JA}[(+)-7-i s o-\mathrm{JA}]$ which is 
assumed to equilibrate to the more stable $(3 R, 7 R)-\mathrm{JA}[(-)-\mathrm{JA}]$.The other two diastereomers, the (3S,7S)-JA and the (3S,7R)-JA do not occur in nature (4). OPDA is known to activate gene transcription independently of being converted to $\mathrm{JA}$ and hence acts as a signalling molecule in its own right (16). Four OPR genes have been identified in Arabidopsis with differing activities towards dia-stereoisomers of OPDA. OPR1 utilizes 9R, 13R-OPDA >>9S,13R-OPD but not the 13S-configured isomers, whereas OPR 2 effectively reduces all 4 isomers including the natural 9S,13S -OPDA (cis-[+]-OPDA). Recent studies with the Arabidopsis mutant OPR3 revealed an independent pathway that uses 4,5-didehydrojasmonate for $\beta$-oxidation and JA synthesis (20). Recent studies with OPR mutants in maize provides strong evidence for their involvement in development and immunity to pathogens and insects and - and may also regulate seed germination in Arabidopsis (21).

The cyclopentanone undergoes three rounds of $\beta$-oxidation to shorten the carboxyterminal carbon chain by six carbons to generate JA (Fig 1c). The acetyl-CoA generated can be used for energy production through the Krebs cycle or else serve as a precursor for many metabolic pathways including fatty acid and terpenoid synthesis. JA (or its active forms-see below) are transported around the plant and have been detected in the phloem and xylem of vascular bundles. The transporter protein has been tentatively identified as GTR1 (22).

\section{Cytoplasmic based reactions (Fig 1d)}

Work on the Arabidopsis JA-insensitive mutant jar1-1 revealed that the active form of jasmonic acid was an amino acid conjugate, principally JA-isoleucine (JA-lle) synthesised in the cytoplasm (Fig 1d) (23). Expression of wild-type JAR1 in transgenic jar1-1 plants restored sensitivity to JA and elevated JA-lle to the same level as in the wild type. Gas chromatography-mass spectrometry (GC-MS) analysis of products generated in vitro by recombinant JAR 1 demonstrated that this enzyme forms JA-amido conjugates with several amino acids, including valine, leucine and phenylalanine but at much lower levels than with isoleucine. Wounding studies with Arabidopsis revealed that JA-lle rapidly accumulates (with 5 mins) in leaves distal to the wound site and precedes the onset of early transcriptional responses and was associated with JAZ (Jasmonate Zim domain) protein degradation (see details under JA receptor). Wound-induced systemic production of JA-lle required the (OPDA) reductase 3 (OPR3) in undamaged responding leaves but not in wounded leaves and was largely dependent on the JA-conjugating enzyme JAR1.

\section{Catabolism of JA-lle}

Two major pathways have been proposed to inactivate JA-lle (24) a) The hydrolysis of the isoleucine moiety from JA-lle catalysed by amidohydrolase (AMH) (Fig. 2). The JA generated can be either hydroxylated to 12-hydroxy-JA, glycosylated, methylated to form methyl jasmonate (Me-JA) or decarboxylated to synthesise cisjasmone (CJ) or b) JA-lle undergoes sequential oxidation of the omega-end of the pentenyl side-chain. These reactions are catalysed by specific members of the cytochrome P450 (CYP) subfamily, CYP94 $(25,26)$. In the first step JA-lle is hydroxylated to 12-hydroxy JA-lle. This then can then either undergo further 
oxidation to produce the 12-carboxy-JA-lle (27) or else can be glycosylated to generate 12-O-glycosyl-JA-Ile (28) (Fig. 2).

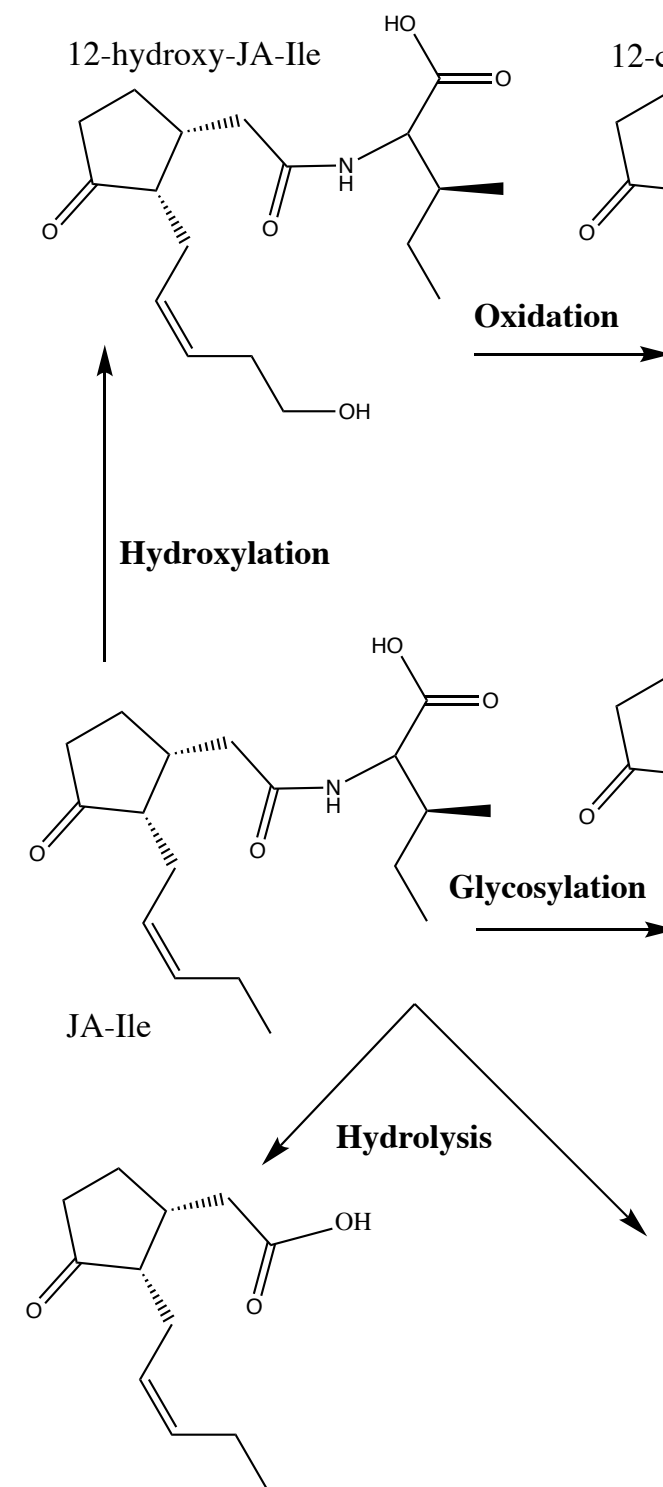

JA

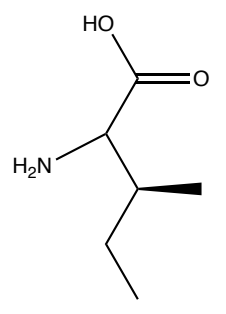

Ile

Fig. 2. Catabolism of Jasmonate-isoleucine.

Jasmonate-isoleucine (JA-lle) can be metabolised through several different routes. It can be hydrolysed into its constituent molecules JA and isoleucine by an amidohydrolase. In addition, hydroxylation by a P450 enzyme yields 12-hydroxy-JA-lle which can be further oxidised by the same class of enzyme to generate the carboxy-derivative. Furthermore, JA-lle can be glycosylated and this could be the transported form of the phytohormone around the plant. 


\section{Conversion to air borne signalling molecules}

\section{Methyl jasmonate}

Methyl jasmonate (Me-JA) is an ester formed from the donation of a methyl group from S-adenosyl-L-methionine (SAM) to JA catalysed by the enzyme SAM:JA carboxyl methyltransferase. Unlike JA, Me-JA is volatile and has been detected emanating from plant leaves during herbivore attack. This molecule can be detected by plants in the vicinity that have not yet been predated and hence it acts as an advanced air borne warning signal (4). One of the common markers for JA pathway activation in tomato and potato is the production of the protease inhibitor, PIN II (29). This acts as an anti-feedant by interfering with the digestion of proteins in the gut of the insect. In turn, the insect gets a lower nutrient value out of the ingested food and hence takes a longer time to complete its life cycle. The consequence of this delay is that inclement weather may intervene and hence reduce the survival of the herbivore. In addition, Me-JA is just one component of a complex mixture of volatiles released during herbivory that can also act as signal cues for the attraction of predators to the herbivore such as parasitic wasps (30).

Transcript analysis following Me-JA treatment is also known to stimulate the production of a range of compounds collectively called phytoalexins, that include terpenoids, glycosteroids and alkaloids, many of which are antimicrobial (31). In maize, Me-JA stimulates carotenoid biosynthesis (32), while in the medicinal plant, Scrophularia striata, it accelerates sucrose degradation and directs the metabolic fluxes towards flavonoids and phenylethanoid glycoside synthesis through a change in enzyme activity at the entry point of the phenylpropanoid pathway (33). MeJA also alters the rate of amino acid biosynthesis and manipulates cell growth accordingly (33).

\section{cis-Jasmone}

cis-Jasmone (CJ) can be synthesised via two routes in plants. In pathway A, 7-iso$\mathrm{JA}(3 R, 7 R)$ is converted to 3,7-didehydrojasmonate $(3,7 \mathrm{DH}-\mathrm{JA})$ which then undergoes decarboxylation to generate CJ. In pathway $\mathrm{B}, 3,7 \mathrm{DH}-\mathrm{JA}$ can also be synthesised via iso-OPDA (34)(Fig 3). 

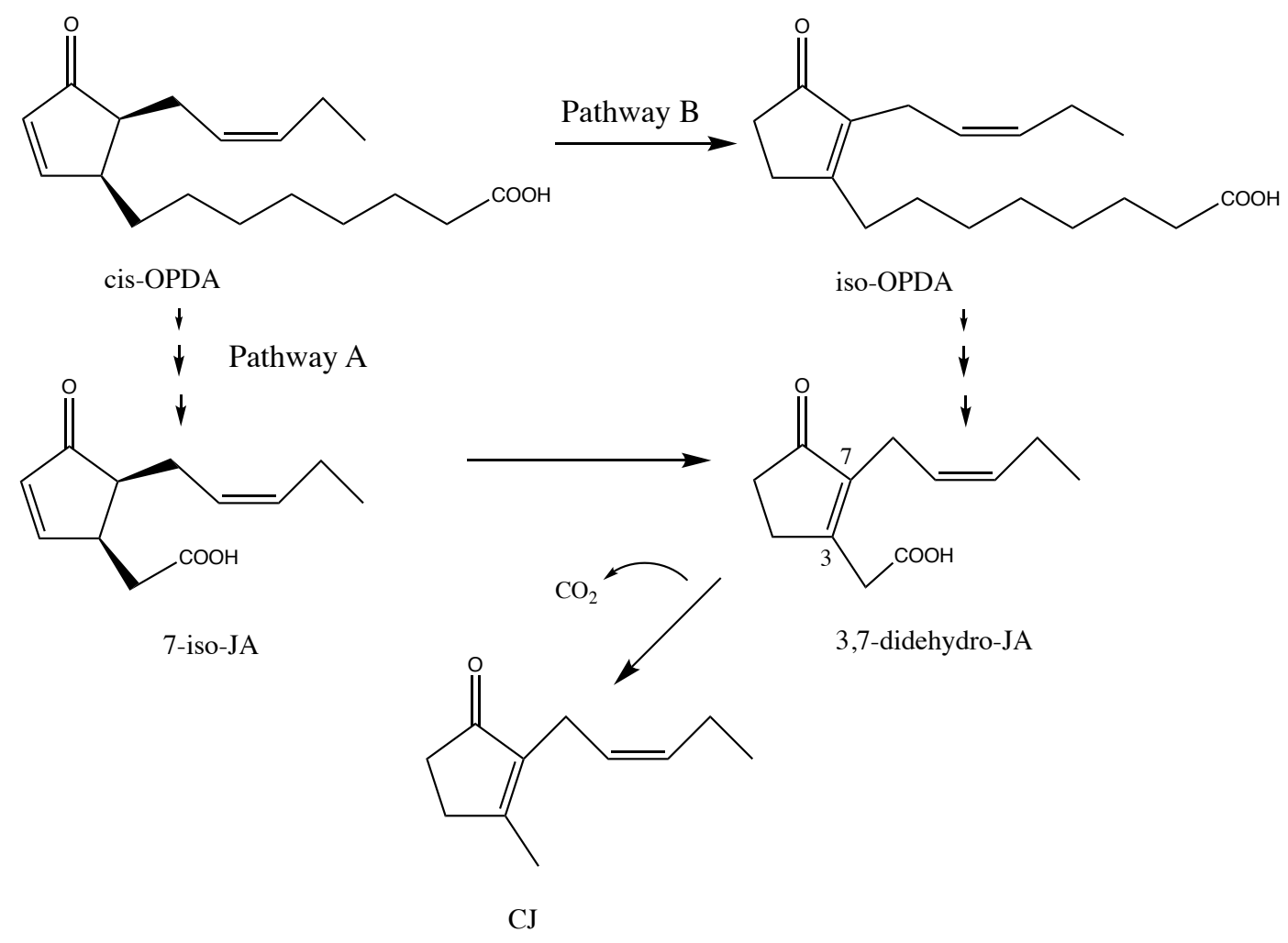

Fig. 3. Biosynthesis of cis-Jasmone.

Two pathways are proposed for the biosynthesis of cis-jasmone. In pathway A, cis-OPDA undergoes $\beta$-oxidation to 7-iso-JA and is then converted to 3,7-didehydro-JA (3,7-DH-JA). In pathway $\mathrm{B}$, the cisOPDA is first converted to iso-OPDA and then metabolised to the 3,7-DH-JA. In both scenarios the 3,7-DH-JA undergoes decarboxylation to generate cis-jasmone (CJ).

$\mathrm{CJ}$ is an important ingredient in the perfume industry and is released from the flowers of many plants such as jasmine, neroli (Citrus bigaradia), jonquil (Narcissus jonquilla L.), bergamot (Citrus bergamia), and members of the Pittosporum family. In terms of plant defense, $\mathrm{CJ}$ is considered to act in a manner similar to JA being released from wounded leaves and acting as an insect repellent and/or an attractant for insect predators (e.g. aphid parasitoids (35). For example, application of CJ to wheat was shown to be particularly effective against cereal aphids and also had deterrent effects on phytophagous thrips species and wheat stem sawflies (30). In tomato plants, $\mathrm{CJ}$ foliar application resulted in an increase production of volatile organic compounds (VOCs) including green leaf volatiles and terpenes with a corresponding increase in the transcript levels for the genes encoding them. In addition, the behaviour of a common tomato pest, Spodoptera exigua, was also influenced in terms of oviposition and egg laying by CJ (36). Increase VOCs were also reported in potato with $(E)$-2-hexenal, $(E, E)-4,8,12$-trimethyl-1,3,7,11tridecatetraene, $(E)$-beta-farnesene, $(E)$-4,8-dimethyl-1,3,7-nonatriene, methyl salicylate, $\mathrm{CJ}$ and methyl benzoate being the main VOCs contributing to aphid behavioural responses (37). In some plants related to chrysanthemum, jasmone can also be converted to jasmolone, a pyrethrin with insecticidal properties, through hydroxylation by cytochrome P450 (38). 


\section{Jasmonate receptor}

In recent years much effort has been devoted to understanding the structure, function and regulation of the JA receptor (39). In common with many cell cycle regulatory receptors and indeed with another plant phytohormone, auxin, the JA receptor is in fact a complex made up of several different domains referred to as the SCF (Skp, Cullin, F-box) complex (Fig. 4).
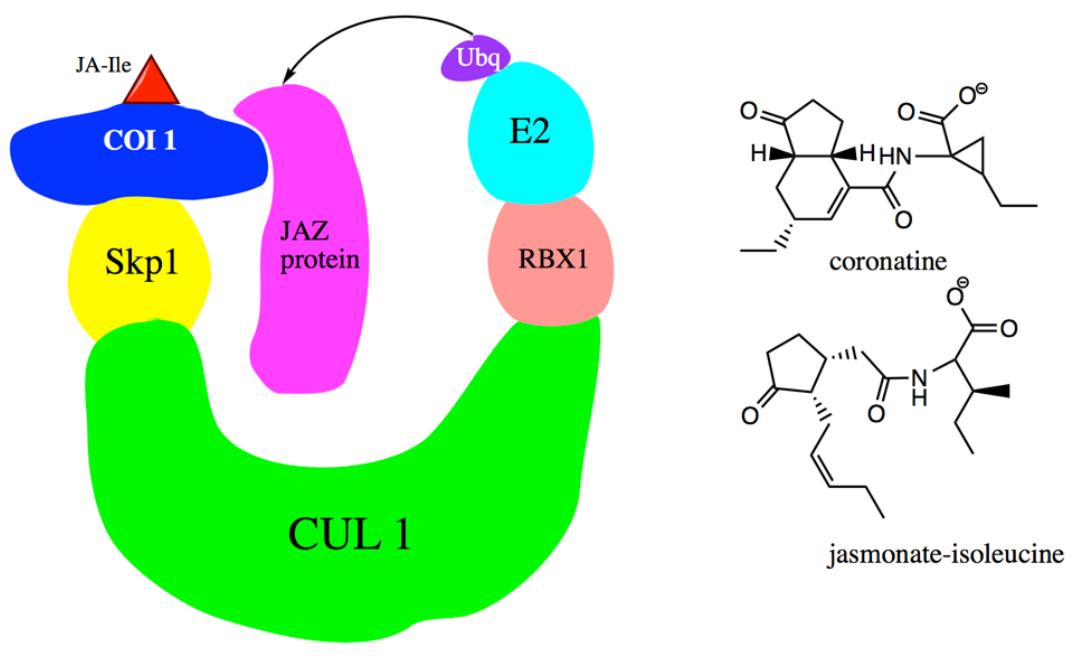

Fig 4. Structure of the SCFCOI1 complex and its inhibitor coronatine.

The SCF complex is composed of several protein domains. It includes the main structural scaffold protein, CUL1 and Skp which is an adaptor protein that binds the F-box domain termed COI 1. RBX 1 contains a zinc finger domain to which the ubiquitinating enzyme, E2, binds. The protein destined for degradation (JAZ) binds to the $\mathrm{COI} 1$ following bind of the JA-lle conjugate and this initiates ubiquitination ( $\mathrm{Ubq})$ by E2. Coronatine is a toxin produced by Pseudomonas syringae and is an analogue of JA-lle used to study JA responses in plants.

The Skp domain (S), is an adaptor protein that is essential for the recognition and binding of F-box proteins. The Cullin domain $(C)$ forms the major structural scaffold of the SCF complex and are a family of hydrophobic scaffold proteins which provide support for ubiquitin ligases. Ubiquitin is a small regulatory protein $(8.6 \mathrm{KDa})$ that is covalently attached to targeted proteins destined for degradation in the $26 \mathrm{~S}$ proteasome. The Cullin domain is also a link between the Skp domain and the Rbx1 domain. Rbx1 domain contains a small, zinc-binding Really Interesting New Gene (RING) finger domain, to which the ubiquitin-conjugating enzyme binds. This binding event allows the transferral of ubiquitin to a lysine residue on the target protein.

Different combinations of Cullin and FBPs can generate hundreds of types of ubiquitin ligases that target different substrates. The F-box domain (FBD) is variable and contributes to the substrate specificity of the SCF complex by first aggregating to 
target proteins independently of the complex. Each FBD may recognize several different substrates in a manner that is dependent on post-translational modifications such as phosphorylation or glycosylation. FBD then binds to Skp domain of the SCF complex using an F-box motif, bringing the target protein into proximity with the functional ubiquitin-conjugating enzyme. In the specific case of JA signalling the Fbox protein is called Coi1(coronatine insensitive). Coronatine is a bacterial toxin and structural analogue of JA.

JA-lle must bind to the the Coi1 of the SCF complex for signal transduction to occur (40).

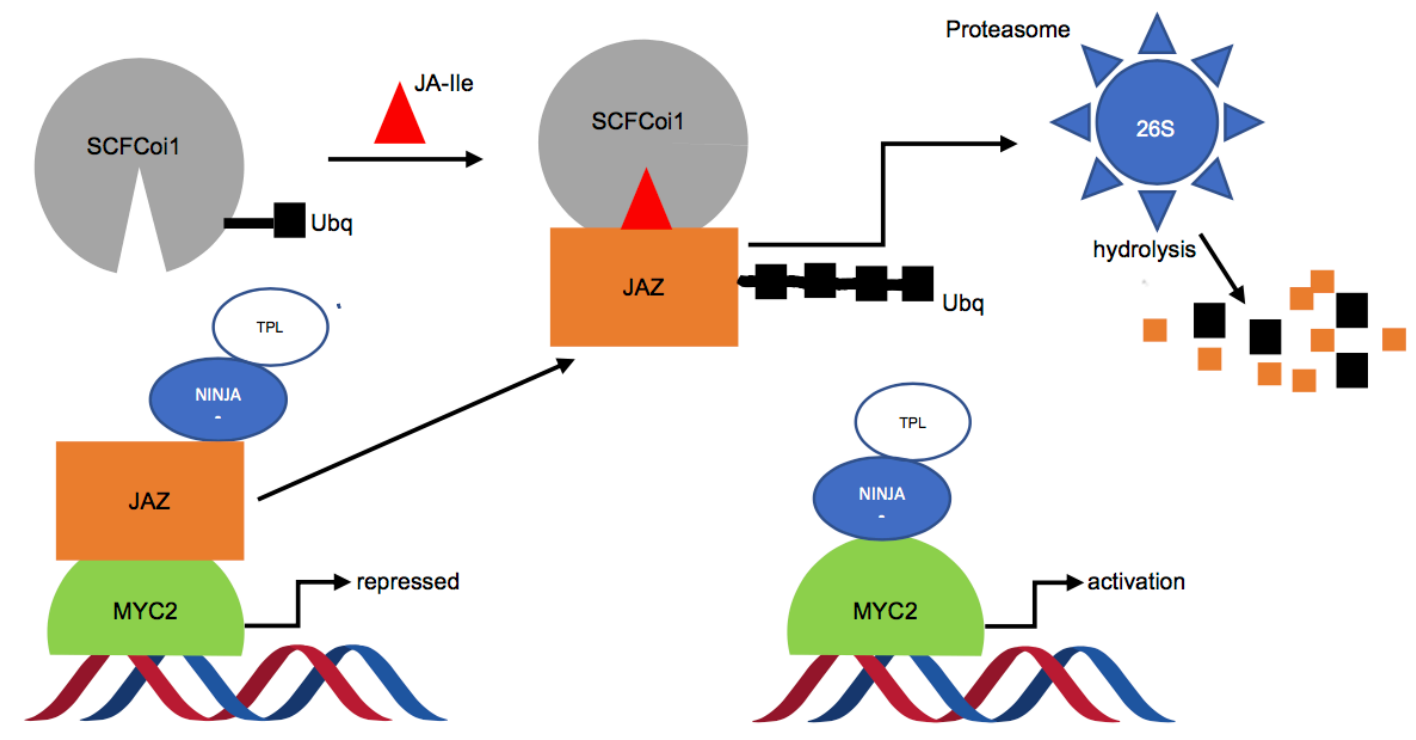

Fig. 5. The Jasmonate-isoleucine receptor and its activation.

In the non-responsive mode (i.e. low levels of the hormone JA-Ile) the genes are repressed by the binding of JAZ proteins to the transcription factor MYC2. Following the synthesis of JA-lle, the receptor, SCFCoi1, binds this ligand and activates the removal of JAZ for ubiquitination (Ubq) which is then targeted to the $26 \mathrm{~S}$ proteasome for hydrolysis. Removal of JAZ results in the activation of JA-Ile responsive genes. TPL is a co-repressor that modulates MYC2 and NINJA is an adaptor protein.

Extensive molecular modelling of the interaction between Coi1 and different jasmonate compounds such as JA, MeJA, OPDA, (+)-7-iso-JA and coronatine, revealed that only (+)-7-iso-JA and coronatine could fit well within the Coi1 surface pocket (41). Both MeJA and JA are too small to fit within the pocket and OPDA could not form a stable interaction with the surface pocket (40). Sensing of JA-lle by Coi1 rapidly triggers genome-wide transcriptional changes that are largely regulated by the basic helix-loop-helix transcription factor (TF) MYC2 (42). When JA-Ile levels are low, MYC2 is repressed by the transcriptional repressors, JASMONATE ZIM (zincfinger protein expressed in the inflorescence meristem) domain proteins (JAZ proteins) (Fig. 4). JAZ proteins comprise a large family of transcriptional repressors that modulate JA responses and belong to a larger family of TIFY proteins (so called 
because of the highly conserved TIF[F/Y] XG motif residing in the ZIM domain of all family members. The number of $J A Z$ genes varies across the Plant Kingdom so for example in lower plants, such as the bryophyte, Marchantia polymorpha, there is one $J A Z$ gene whereas there are more than 20 in many higher plants. The Arabidopsis genome encodes $13 \mathrm{JAZ}$ proteins that are classified into 5 phylogenic groups. Recent work has revealed that additional proteins are involved in signal transduction and include an additional co-repressor, TPL and an adaptor protein termed NINJA (novel interactor of JAZ)(43). Following binding of JA-Ile to SCFCoi1, JAZ proteins are targeted for ubiquitination (Ubq) (Fig. 5). The JAZ-Ubq proteins are then hydrolysed by the $26 \mathrm{~S}$ proteasome. The degradation of the JAZ protein allows the MYC2 transcription factor to facilitate the JA response through gene expression (44).

In tomato, 655 MYC2-targeted JA-responsive genes have been identified and these genes are highly enriched in Gene Ontology categories related to TFs and the early response to JA, indicating that MYC2 functions at a high hierarchical level to regulate JA-mediated gene transcription (42). In Arabidopsis JAZ genes have been shown to be important in regulating plant growth which is often compromised following pathogen/pest attack. Indeed a higher-order mutant (termed jazD) defective in 10 JAZ genes (JAZ1-7, -9, -10, and -13) exhibited robust resistance to insect herbivores and fungal pathogens, but was accompanied by slow vegetative growth and poor reproductive performance (45). JAZ proteins promote growth and reproductive success at least in part by preventing catastrophic metabolic effects of an unrestrained immune response. In studies using Arabidopsis challenged with the generalist insect herbivore Spodoptera exigua it was shown that most members of the JAZ gene family were highly expressed with transcript levels increasing within 5 min of tissue damage, coincident with a large (approximately 25-fold) rise in JA and JA-lle levels (46). JAZs, MYC2, and genes encoding several JA biosynthetic enzymes are primary response genes whose expression is derepressed upon COI1dependent turnover of a labile repressor protein(s). Recent work on the jaz4-1 mutant of Arabidopsis has shown that it is hyper-susceptible to Pseudomonas syringae while Arabidopsis lines overexpressing a JAZ4 protein have enhanced resistance to this bacterium (47). For a recent review focusing in-depth on the JA signal transduction pathway and its regulatory elements the reader is referred to (48).

\section{Cross talk: JA interactions with other signalling pathways}

Plant responses to developmental and environmental cues are under the control of multiple phytohormones. These include, amongst others, auxins, gibberellins, abscisic acid, ethylene, cytokinins, brassinosteroids, salicylic acid, strigolactones, polyamines and jasmonic acid. It is evident, therefore, that a co-ordinated plant response to any given signal must, of necessity, involve multiple signalling pathways and hence extensive cross talk between these pathways must exist (49). For example, plants under biotic attack will divert resources away from growth and into the synthesis of defensive compounds (50). Similarly, plants under abiotic stress will prioritise their response to that cue, e.g. there is a functional convergence of oxylipin and abscisic acid pathways in the control of stomatal closure in response to drought in Arabidopsis, tomato and rapeseed (51). This is regulated at the level of OPDA, 
with plants producing higher OPDA levels exhibiting enhanced drought tolerance and reduced stomatal aperture that acts in conjunction with $A B A$ to bring about the response (51). Similarly, JA-amino acid conjugate levels can be controlled by enzymes that catabolise auxin-amino acid conjugates and represents an example of a metabolic-level crosstalk between the auxin and JA signaling pathways (52). In Arabidopsis, OPR3 functions to down-regulate primary root growth under phosphorus limitation and interacts with ethylene and gibberellin signalling pathways to co-ordinate this response (53). Interestingly, JA also conjugates to the ethylene precursor, 1-aminocyclopropane-1-carboxylic acid (ACC) and this suggests coregulation of JA and ethylene signalling pathways.

It appears that JAZ proteins constitute a major hub for crosstalk with diverse stress and developmental signalling pathways that may serve to optimise plant survival in changing environments (48). These nodes of signal interaction modulate jasmonate signaling outputs by mechanisms tied to changes in JAZ abundance or accessibility (48). JAZ protein families have been associated with activation of particular pathways in response to biotic stress (e.g. herbivory), developmental responses (e.g. growth inhibition) and abiotic stress (e.g. drought) (Fig. 6). Thus, fluctuating levels of JA-lle sensed by combinatorial assemblies of COI1-JAZ coreceptors may serve not only to alter the transcriptional state of genes bound by JAZ-transcription factor complexes but are also likely to mediate crosstalk between pathways by propagating changes in a broader protein-protein interaction network (48). 


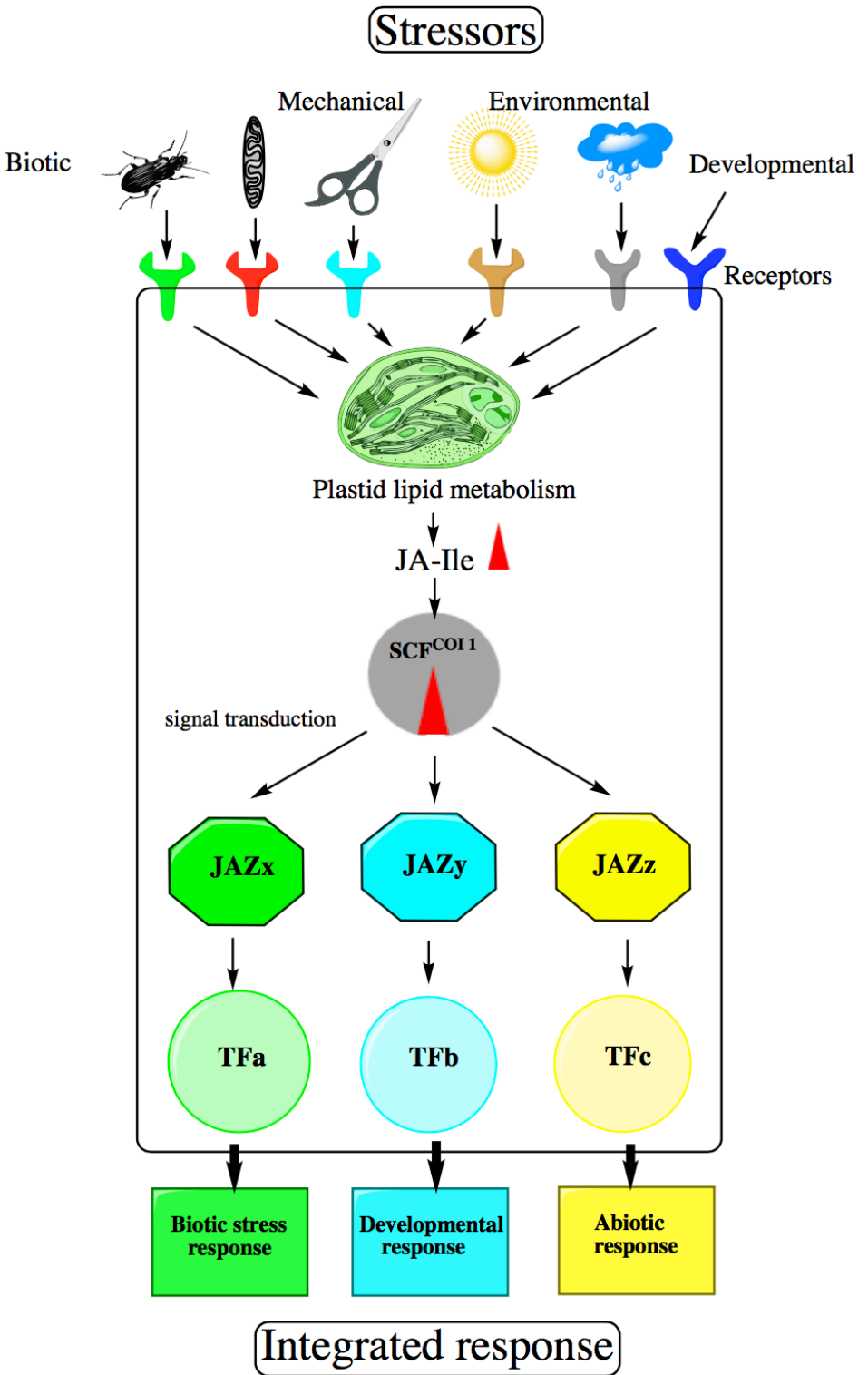

Fig. 6. Stress signal inputs and integrated output responses to the JA-Ile signalling pathway.

Inductive signals include biotic stress from microbe and herbivore attack, endogenous developmental signals and abiotic stress from mechanical damage and other environmental cues such as drought and salinity. These signals are recognised by receptors at the cell surface to trigger de novo synthesis of JA-lle from plastid lipids. JA-lle promotes degradation of multiple JAZ proteins (exemplified by $J A Z x-z$ ) via the action of the $E 3$ ubiquitin ligase SCFCOI1 and the $26 S$ proteasome. Degradation of $J A Z$ relieves repression on the JAZ-interacting transcription factors (exemplified by TFa-c) that govern various physiological output responses involved in growth, development, and tolerance to biotic and abiotic stresses. Many JAZ proteins functionally interact with multiple transcription factors. 


\section{Future}

Clearly great progress has been made on establishing the role of JAs in plant biology in recent years and some surprising functions have been revealed through the use of mutants that could not have been logically anticipated. The current focus of attention is on deciphering the roles of the JAZ hubs in co-ordinating plant responses to JAs and to establish how these hubs interact with other plant hormone signalling pathways to co-ordinate plant responses. While the focus has been on JA-lle, the receptors for other bioactive JAs such as Me-JA and CJ are lagging behind. Do they use closely related SCFCoi receptors to that used by JA-Ile? In a world with over 7 billion people the challenge will remain as to how we can produce sufficient food to sustain population growth. With estimates of $20-30 \%$ of food production lost annually to pests and pathogens our ability to combat this problem will become more pressing. Hence our ability to fundamentally understand the control of plant responses to these biotic challenges whilst simultaneously maintaining yields is of paramount importance.

\section{Summary points}

- Understanding the defensive capabilities of plants towards pests and pathogens is necessary if we are going to develop crops in the future with enhanced resilience and improved yield performance.

- The role of jasmonates has been well established as a major contributor to plant biotic defense. The biosynthetic pathways are largely established and the genes cloned. One of the key receptors for jasmonate is the SCFCoi1, a ubiquitin ligase complex that leads to the degradation of JAZ repressor proteins that in turn leads to gene activation.

- Understanding how these JAZ protein hubs interact with other phytohormones in controlling plant responses, often conflicting, such as metabolic investment in growth versus the synthesis of defensive compounds is at the forefront of current research activity.

\section{References}

1. Wasternack C, Strnad M. Jasmonates: News on Occurrence, Biosynthesis, Metabolism and Action of an Ancient Group of Signaling Compounds. International Journal of Molecular Sciences. 2018;19(9).

2. Howe GA, Yoshida Y. Evolutionary Origin of JAZ Proteins and Jasmonate Signaling. Molecular Plant. 2019;12(2):153-5.

3. Griffiths G. Biosynthesis and analysis of plant oxylipins. Free Radical Research. 2015;49(5):565-82.

4. Wasternack C, Feussner I. The Oxylipin Pathways: Biochemistry and Function. In: Merchant SS, editor. Annual Review of Plant Biology, Vol 69. Annual Review of Plant Biology. 692018. p. 36386.

5. Vicente J, Cascon T, Vicedo B, Garcia-Agustin P, Hamberg M, Castresana C. Role of 9Lipoxygenase and alpha-Dioxygenase Oxylipin Pathways as Modulators of Local and Systemic Defense. Molecular Plant. 2012;5(4):914-28. 
6. Nilsson AK, Fahlberg $\mathrm{P}$, Johansson ON, Hamberg M, Andersson MX, Ellerstrom M. The activity of HYDROPEROXIDE LYASE 1 regulates accumulation of galactolipids containing 12-oxophytodienoic acid in Arabidopsis. Journal of Experimental Botany. 2016;67(17):5133-44.

7. Itoh A, Howe GA. Molecular cloning of a divinyl ether synthase - Identification as a CYP74 cytochrome P-450. Journal of Biological Chemistry. 2001;276(5):3620-7.

8. Prost I, Dhondt S, Rothe G, Vicente J, Rodriguez MJ, Kift N, et al. Evaluation of the antimicrobial activities of plant oxylipins supports their involvement in defense against pathogens. Plant Physiology. 2005;139(4):1902-13.

9. Furstenberg-Hagg J, Zagrobelny M, Bak S. Plant Defense against Insect Herbivores. International Journal of Molecular Sciences. 2013;14(5):10242-97.

10. Zhang L, Zhang F, Melotto M, Yao J, He SY. Jasmonate signaling and manipulation by pathogens and insects. Journal of Experimental Botany. 2017;68(6):1371-85.

11. Wang K, Guo Q, Froehlich JE, Hersh HL, Zienkiewicz A, Howe GA, et al. Two Abscisic AcidResponsive Plastid Lipase Genes Involved in Jasmonic Acid Biosynthesis in Arabidopsis thaliana (vol 30, pg 1006, 2018). Plant Cell. 2019;31(11):2805-.

12. Zhang M, Demeshko Y, Dumbur R, Iven T, Feussner I, Lebedov G, et al. Elevated -Linolenic Acid Content in Extra-plastidial Membranes of Tomato Accelerates Wound-Induced Jasmonate Generation and Improves Tolerance to the Herbivorous Insects Heliothis peltigera and Spodoptera littoralis. Journal of Plant Growth Regulation. 2019;38(2):723-38.

13. Stenzel I, Otto M, Delker C, Kirmse N, Schmidt D, Miersch O, et al. ALLENE OXIDE CYCLASE (AOC) gene family members of Arabidopsis thaliana: tissue- and organ-specific promoter activities and in vivo heteromerization. J Exp Bot. 2012;63(17):6125-38.

14. Ibrahim A, Schutz AL, Galano JM, Herrfurth C, Feussner K, Durand T, et al. The alphabet of galactolipids in Arabidopsis thaliana. Front Plant Sci. 2011;2.

15. Andersson MX, Hamberg M, Kourtchenko O, Brunnstrom A, McPhail KL, Gerwick WH, et al. Oxylipin profiling of the hypersensitive response in Arabidopsis thaliana - Formation of a novel oxophytodienoic acid-containing galactolipid, arabidopside E. J Biol Chem. 2006;281(42):31528-37.

16. Taki N, Sasaki-Sekimoto Y, Obayashi T, Kikuta A, Kobayashi K, Ainai T, et al. 12-oxophytodienoic acid triggers expression of a distinct set of genes and plays a role in wound-induced gene expression in Arabidopsis. Plant Physiol. 2005;139(3):1268-83.

17. Mosblech A, Feussner I, Heilmann I. Oxylipins: Structurally diverse metabolites from fatty acid oxidation. Plant Physiol Biochem. 2009;47(6):511-7.

18. Zhou Y, Behrendt J, Sutherland AJ, Griffiths G. Synthetic molecular mimics of naturally occurring cyclopentenones exhibit antifungal activity towards pathogenic fungi. Microbiology-Sgm. 2011;157:3435-45.

19. Nguyen CT, Martinoia E, Farmer EE. Emerging Jasmonate Transporters. Molecular Plant. 2017;10(5):659-61.

20. Chini A, Monte I, Zamarreno AM, Hamberg M, Lassueur S, Reymond P, et al. An OPR3independent pathway uses 4,5-didehydrojasmonate for jasmonate synthesis. Nature Chemical Biology. 2018;14(2):171-+.

21. Dave A, Hernandez ML, He ZS, Andriotis VME, Vaistij FE, Larson TR, et al. 12-OxoPhytodienoic Acid Accumulation during Seed Development Represses Seed Germination in Arabidopsis. Plant Cell. 2011;23(2):583-99.

22. Ishimaru Y, Oikawa T, Suzuki T, Takeishi S, Matsuura H, Takahashi K, et al. GTR1 is a jasmonic acid and jasmonoyl-L-isoleucine transporter in Arabidopsis thaliana. Bioscience Biotechnology and Biochemistry. 2017;81(2):249-55.

23. Staswick PE, Tiryaki I. The oxylipin signal jasmonic acid is activated by an enzyme that conjugates it to isoleucine in Arabidopsis. Plant Cell. 2004;16(8):2117-27.

24. Bruckhoff V, Haroth S, Feussner K, Konig S, Brodhun F, Feussner I. Functional Characterization of CYP94-Genes and Identification of a Novel Jasmonate Catabolite in Flowers. Plos One. 2016;11(7). 
25. Kitaoka N, Kawaide H, Amano N, Matsubara T, Nabeta K, Takahashi K, et al. CYP94B3 activity against jasmonic acid amino acid conjugates and the elucidation of 12-0-beta-glucopyranosyljasmonoyl-L-isoleucine as an additional metabolite. Phytochemistry. 2014;99:6-13.

26. Heitz T, Widemann E, Lugan R, Miesch L, Ullmann P, Desaubry L, et al. Cytochromes P450 CYP94C1 and CYP94B3 Catalyze Two Successive Oxidation Steps of Plant Hormone Jasmonoylisoleucine for Catabolic Turnover. Journal of Biological Chemistry. 2012;287(9):6296-306.

27. Koo AJ, Thireault C, Zemelis S, Poudel AN, Zhang T, Kitaoka N, et al. Endoplasmic Reticulumassociated Inactivation of the Hormone Jasmonoyl-L-Isoleucine by Multiple Members of the Cytochrome P450 94 Family in Arabidopsis. Journal of Biological Chemistry. 2014;289(43):29728-38. 28. Haroth S, Feussner K, Kelly AA, Zienkiewicz K, Shaikhqasem A, Herrfurth C, et al. The glycosyltransferase UGT76E1 significantly contributes to 12-0-glucopyranosyl-jasmonic acid formation in wounded Arabidopsis thaliana leaves. Journal of Biological Chemistry. 2019;294(25):9858-72.

29. Singh S, Singh A, Kumar S, Mittal P, Singh IK. Protease inhibitors: recent advancement in its usage as a potential biocontrol agent for insect pest management. Insect Science. 2020;27(2):186201.

30. Bayram A, Tonga A. cis-Jasmone treatments affect pests and beneficial insects of wheat (Triticum aestivum L.): the influence of doses and plant growth stages. Crop Protection. 2018;105:70-9.

31. Shi J, Ma CY, Qi DD, Lv HP, Yang T, Peng QH, et al. Transcriptional responses and flavor volatiles biosynthesis in methyl jasmonate-treated tea leaves. Bmc Plant Biology. 2015;15.

32. Luo H, He WW, Li DJ, Bao YH, Riaz A, Xiao YD, et al. Effect of methyl jasmonate on carotenoids biosynthesis in germinated maize kernels. Food Chemistry. 2020;307.

33. Sadeghnezhad E, Sharifi M, Zare-Maivan H, Chashmi NA. Time-dependent behavior of phenylpropanoid pathway in response to methyl jasmonate in Scrophularia striata cell cultures. Plant Cell Reports. 2020;39(2):227-43.

34. Dabrowska P, Boland W. iso-OPDA: An early precursor of cis-jasmone in plants? Chembiochem. 2007;8(18):2281-5.

35. Matthes MC, Bruce TJA, Ton J, Verrier PJ, Pickett JA, Napier JA. The transcriptome of cisjasmone-induced resistance in Arabidopsis thaliana and its role in indirect defence. Planta. 2010;232(5):1163-80.

36. Disi JO, Zebelo S, Ngumbi E, Fadamiro HY. cis-Jasmone primes defense pathways in tomato via emission of volatile organic compounds and regulation of genes with consequences for Spodoptera exigua oviposition. Arthropod-Plant Interactions. 2017;11(4):591-602.

37. Sobhy IS, Woodcock CM, Powers SJ, Caulfield JC, Pickett JA, Birkett MA. cis-Jasmone Elicits Aphid-Induced Stress Signalling in Potatoes. Journal of Chemical Ecology. 2017;43(1):39-52.

38. Li W, Zhou F, Pichersky E. Jasmone Hydroxylase, a Key Enzyme in the Synthesis of the Alcohol Moiety of Pyrethrin Insecticides. Plant Physiology. 2018;177(4):1498-509.

39. Wasternack C, Song SS. Jasmonates: biosynthesis, metabolism, and signaling by proteins activating and repressing transcription. Journal of Experimental Botany. 2017;68(6):1303-21. 40. Yan JB, Zhang C, Gu M, Bai ZY, Zhang WG, Qi TC, et al. The Arabidopsis CORONATINE INSENSITIVE1 Protein Is a Jasmonate Receptor. Plant Cell. 2009;21(8):2220-36.

41. Sheard LB, Tan X, Mao HB, Withers J, Ben-Nissan G, Hinds TR, et al. Jasmonate perception by inositol-phosphate-potentiated COI1-JAZ co-receptor. Nature. 2010;468(7322):400-U301.

42. Du MM, Zhao JH, Tzeng DTW, Liu YY, Deng L, Yang TX, et al. MYC2 Orchestrates a Hierarchical Transcriptional Cascade That Regulates Jasmonate-Mediated Plant Immunity in Tomato. Plant Cell. 2017;29(8):1883-906.

43. Pauwels L, Barbero GF, Geerinck J, Tilleman S, Grunewald W, Perez AC, et al. NINJA connects the co-repressor TOPLESS to jasmonate signalling. Nature. 2010;464(7289):788-U169. 
44. An CP, Li L, Zhai QZ, You YR, Deng L, Wu FM, et al. Mediator subunit MED25 links the jasmonate receptor to transcriptionally active chromatin. Proceedings of the National Academy of Sciences of the United States of America. 2017;114(42):E8930-E9.

45. Guo Q, Yoshida Y, Major IT, Wang K, Sugimoto K, Kapali G, et al. JAZ repressors of metabolic defense promote growth and reproductive fitness in Arabidopsis. Proceedings of the National Academy of Sciences of the United States of America. 2018;115(45):E10768-E77.

46. Chung HS, Koo AJK, Gao XL, Jayanty S, Thines B, Jones AD, et al. Regulation and function of Arabidopsis JASMONATE ZIM-domain genes in response to wounding and herbivory. Plant Physiology. 2008;146(3):952-64.

47. Oblessuc PR, Obulareddy N, DeMott L, Matiolli CC, Thompson BK, Melotto M. JAZ4 is involved in plant defense, growth, and development in Arabidopsis. Plant Journal. 2020;101(2):37183.

48. Howe GA, Major IT, Koo AJ. Modularity in Jasmonate Signaling for Multistress Resilience. In: Merchant SS, editor. Annual Review of Plant Biology, Vol 69. Annual Review of Plant Biology. 692018. p. 387-415.

49. Xu P, Zhao PX, Cai XT, Mao JL, Miao ZQ, Xiang CB. Integration of Jasmonic Acid and Ethylene Into Auxin Signaling in Root Development. Frontiers in Plant Science. 2020;11.

50. Guo Q, Major IT, Howe GA. Resolution of growth-defense conflict: mechanistic insights from jasmonate signaling. Current Opinion in Plant Biology. 2018;44:72-81.

51. Savchenko T, Kolla VA, Wang CQ, Nasafi Z, Hicks DR, Phadungchob B, et al. Functional Convergence of Oxylipin and Abscisic Acid Pathways Controls Stomatal Closure in Response to Drought. Plant Physiology. 2014;164(3):1151-60.

52. Lee HY, Chen YC, Kieber JJ, Yoon GM. Regulation of the turnover of ACC synthases by phytohormones and heterodimerization in Arabidopsis. Plant Journal. 2017;91(3):491-504.

53. Zheng HY, Pan XY, Deng YX, Wu HM, Liu P, Li XX. AtOPR3 specifically inhibits primary root growth in Arabidopsis under phosphate deficiency. Scientific Reports. 2016;6. 